\title{
Levantamento da fauna de lagartos da Fazenda Tepequém, Amajari, Roraima
}

\author{
Karla Janine Rodrigues ${ }^{1}$, Kamila Freitas Moulaz ${ }^{2}$; Tayná Maria Silva Violi ${ }^{3}$; Eliza \\ Ribeiro Costa ${ }^{4}$ \\ 1,2,3 Faculdades Cathedral, Curso de Graduação em Ciências Biológicas, Av. Luís Canúto Chaves, 293, Boa Vista - \\ RR,69307-053; E-mail: biokjrr@gmail.com¹; kamila991634203@gmail.com² ${ }^{2}$ taynavioli@ hotmail.com³ \\ ${ }^{4}$ Centro Universitário Estácio da Amazônia, Centro de Ciências e Saúde, Rua Jornalista Humberto Silva, 308, Boa \\ Vista - RR, 69313-792; e-mail: eliza_ribeiro@yahoo.com.br
}

Recebido em: 26 de março de 2018. Aceito em: 14 de agosto de 2018. Publicado PDF em: 17 de agosto de 2018

\section{RESUMO}

Levantamento da Fauna de lagartos da Fazenda Tepequém, Amajari, Roraima. Os inventários de fauna de lagartos ainda são consideravelmente incompletos ou até inexistentes em algumas regiões tanto do estado de Roraima, quanto na região amazônica. Lagartos são bons modelos para análise de degradação ambiental, tendo em vista que algumas espécies são sensíveis às alterações ambientais e algumas utilizam habitats específicos. Através de armadilhas de interceptação e queda foi possível inventariar os lagartos da Fazenda Tepequém - Amajari, interior de Roraima de abril a outubro de 2017. A fauna de lagartos encontrada é semelhante a outros habitats amazônicos como nos Estados do Pará, Acre, Rondônia e Amazonas.

PALAVRAS CHAVE: Amazônia, Pitfall, Squamata.

\section{ABSTRACT}

Survey of Fauna of Lizards of Tepequém Farm, Amajari, Roraima State. Lizard wildlife list are still considerably incomplete or even nonexistent in some regions of both the state of Roraima and the Amazon region. Lizards are good models for the evaluation of environmental degradation, considering that some species are sensitive to environmental changes and use specific habitats. Through pitfall traps, a lizard inventory was conducted at Tepequém Farm - Amajari, county of Roraima from april until october 2017. The composition of lizards is similar to those found in other Amazonian habitats such as Pará, Acre, Rondônia and Amazonas states.

KEY WORDS: Amazon, Pitfall, Squamata.

\section{INTRODUÇÃO}

A região de Roraima tem vegetação composta por áreas abertas e Byrsonima spp.; as ciperáceas Bulbostylis spp. fechadas, as quais dão identidade regional e e várias espécies de gramíneas, uma delas condicionam a presença de fauna e flora predominando, dependendo dos solos; as áreas adaptadas a estes ambientes (Carvalho 2009). francamente abertas de lavrado, com poucos

Em suas fitofisionomia, as áreas abertas de arbustos; os lagos do lavrado; presença de Roraima apresentam mata de galeria, que são cactáceas, por exemplo, o mandacaru Cereus as matas das margens dos rios de pequeno sp., geralmente associadas a áreas de porte, que atravessam o lavrado; os buritizais, cupinzeiros Cornitermes, os quais também formados por buritis Mauritia flexuosa; as ilhas ocorrem onde os cactos não estão presentes; as de mata, que são manchas de vegetação mais serras em várias regiões do lavrado, onde estão 
presentes no entorno árvores baixas, arvoretas e arbustos (Carvalho \& Carvalho 2015).

A fauna de áreas abertas constitui-se de vários grupos altamente especializados quanto aos fatores climáticos e ambientais e a precariedade dos recursos disponíveis para a sua sobrevivência, e entender essas questões nos levam ao âmago da complexidade de nicho ecológico, o qual engloba sistematicamente a soma de todas as atividades de uma determinada espécie ou indivíduo (Nascimento 2004).

Levando em consideração o número reduzido de pesquisas realizadas na região, o presente trabalho propõe-se a determinar a biodiversidade faunística de lagartos de maneira que possa contribuir para o entendimento da dinâmica comportamental e a preservação desses animais e do ambiente, que frequentemente são ameaçados pela atividade humana.

\section{MATERIAL E MÉTODOS}

O estudo foi realizado entre abril e outubro de 2017, realizando-se 07 (sete) visitas, durante três dias de cada mês.

A primeira localidade amostral

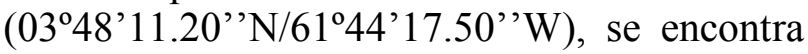
próximo a um barranco, o solo é argiloso e no período chuvoso permanece encharcado, devido a aproximação com um lago de água permanente. Há presença de árvores de médio porte, que sombreiam toda a região onde estão dispostas as armadilhas. Ambas permanecem o dia todo na sombra feita pelas árvores próximas e logo à frente iniciam-se a subida de um pequeno morro.

A segunda localidade amostral

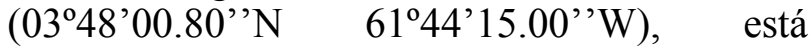
localizada em uma região de pouca vegetação, também próximo a um barranco, o que assemelha a uma voçoroca. Há no entorno das armadilhas presença de plantas como samambaias do campo (Pteridium aquilinum (L.) Kuhn), gramíneas de pequeno porte e Curatella americana L. (caimbé). O solo é arenoso, mas há presença de solos argilosos nos arredores.

A terceira localidade amostral $\left(03^{\circ} 47^{\prime} 93.90^{\prime \prime} \mathrm{N} / 61^{\circ} 44^{\prime} 27.00^{\prime \prime} \mathrm{W}\right) \quad$ está localizada em uma região íngreme, próxima a uma mata de galeria. Apresenta um solo argiloso. Também há presença de gramíneas de tamanhos variados, plantas de pequeno porte como Curatella americana L (caimbé).

Foi utilizado armadilhas de interseção e queda, com baldes 60 litros, cinco baldes para cada localidade amostral, totalizando quinze baldes, todos enterrados no solo de modo a acompanhar as possíveis declividades do ambiente como sugerem (Cechin \& Martins 2000), e em forma de Y, interconectados com

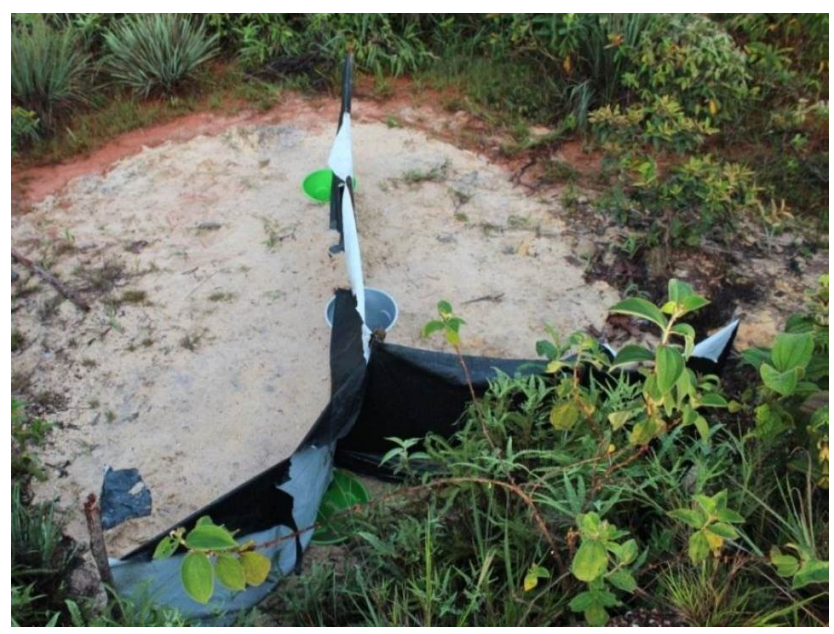

Figura 1. Disposição das armadilhas no solo.

cerca guia de lona preta (Figura 1).

$\mathrm{O}$ estudo também foi realizado com o transecto de amostragem visual (censo), realizado por três pessoas durante o dia, as quais percorrem a pé por uma 01h:20m (uma hora e vinte minutos), em cada dia de campo, totalizando um esforço de quatro horas por módulo em cada incursão a campo.

Durante os censos, foram registrados todos os indivíduos avistados, sendo os mesmos procurados em rochas, revolvendo-se o folhiços, troncos caídos e na margem de corpos d'água, quando presentes (Silva \& Rossa-Feres, 2007). Para que não houvesse recontagem de indivíduos ao longo do período de amostragem, os indivíduos capturados excedentes a 8 exemplares por espécie, foram marcados individualmente por amputação dos dedos e artelhos seguindo o método Donnelly (1994) adaptado para lagartos. Os espécimes de identificação duvidosa eram coletados e eutanasiados para serem comparados com espécimes depositados em coleções e consulta a especialistas, de forma a realizar a determinação inequívoca da espécie.

A eutanásia foi realizada através da administração de anestésico (lidocaína) via injeção intraperitoneal (Freitas et al. 2017). Os espécimes coletados eram pesados, medidos, 
etiquetados e preservados em formol a $10 \%$, durante 12 horas, sendo posteriormente acondicionados em álcool a 70\%, para ser depositado como material testemunho e para auxiliar na identificação das espécies nos módulos de amostragem. Todos os animais coletados foram depositados na Coleção Zoológica do Laboratório de Zoologia da Universidade Federal de Roraima (LABZOA) (Apêndice 01). A coleta e transporte do material foi feito sob autorização do ICMBio/ Sisbio número 57213-1.

\section{RESULTADOS E DISCUSSÃO}

Foram registrados 113 espécimes de lagartos distribuídos em três famílias: Teiidae (10), Gekkonidae (12), e Tropiduridae (91). Destes, foram encontrados Ameiva ameiva (10); Hemidactylus palaichthus (12); Tropidurus hispidus (91) espécimes. Os animais foram encontrados em ambientes variados, desde mata de galeria, ambientes próximos a cachoeiras, áreas de morro, próximos a lagos, campos abertos, com presença de gramíneas e próximo à rochas. A maioria dos espécimes $(\mathrm{N}$ $=91, \mathrm{~N} \%=80,5 \%$ ), foi registrada com o uso de metodologia de busca ativa. A espécie mais representativa $(\mathrm{N}=6, \mathrm{~N} \%=9,7 \%)$ nas armadilhas de interseção e queda foi o teídeo Ameiva ameiva.

As armadilhas de interseção e queda registraram duas espécies de duas famílias diferentes, Tropidurus hispidus e Ameiva ameiva, com variação de idade (adulto e juvenil) para ambas espécies. Na busca ativa, Tropidurus hispidus foi registrada com mais frequência (91 espécimes).

As espécies registradas no presente estudo também são encontradas nos principais habitats amazônicos. A presença das três famílias já foi registrada desde o ano de 1998, quando Nascimento (1998) descreveu a ocorrência delas no lavrado de Roraima. Macedo et al. (2008) registraram a presença de ambas as famílias em um levantamento de fauna de lagartos em áreas de floresta e de pastagem em Espigão do Oeste, estado de Rondônia, sudoeste da Amazônia.

Monteiro et al. (2010), também registraram a presença das famílias Teiidae e Iguanidae na Floresta Nacional do Tapirapé - Aquiri, sudeste do Pará. Mendes-Pinto \& Tello (2010) descreveram a presença das duas famílias

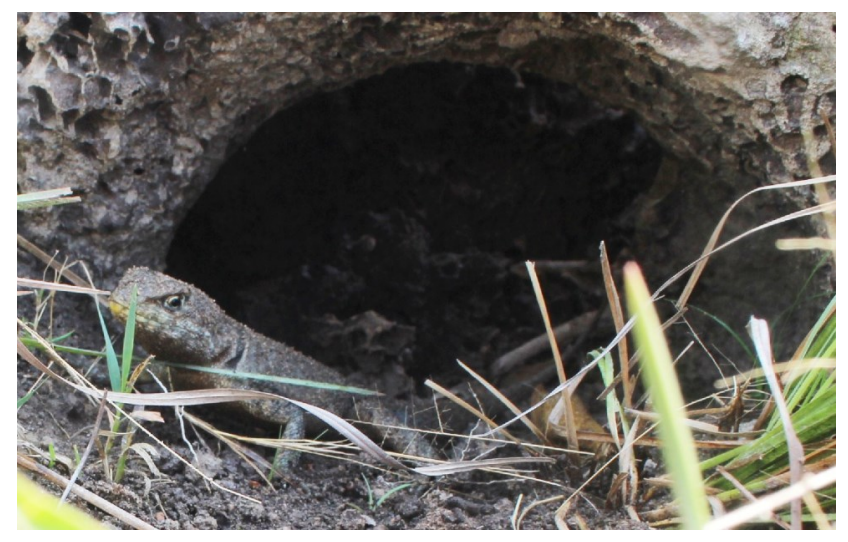

Figura 2. Registro de Tropidurus hispidus próximo a um cupinzeiro.

acima citadas em uma região do oeste do Pará, e afirmam que ambas são comuns na região amazônica e possuem ampla distribuição, ocorrendo também nas regiões de Savana. Algumas espécies de ampla distribuição presente no lavrado de Roraima tem hoje populações distribuídas de várias formas no domínio amazônico (Carvalho 2009).

Tropidurus hispidus foi comumente encontrado em regiões variadas, desde próximo a casas, próximo a cupinzeiros (Figura 2), em

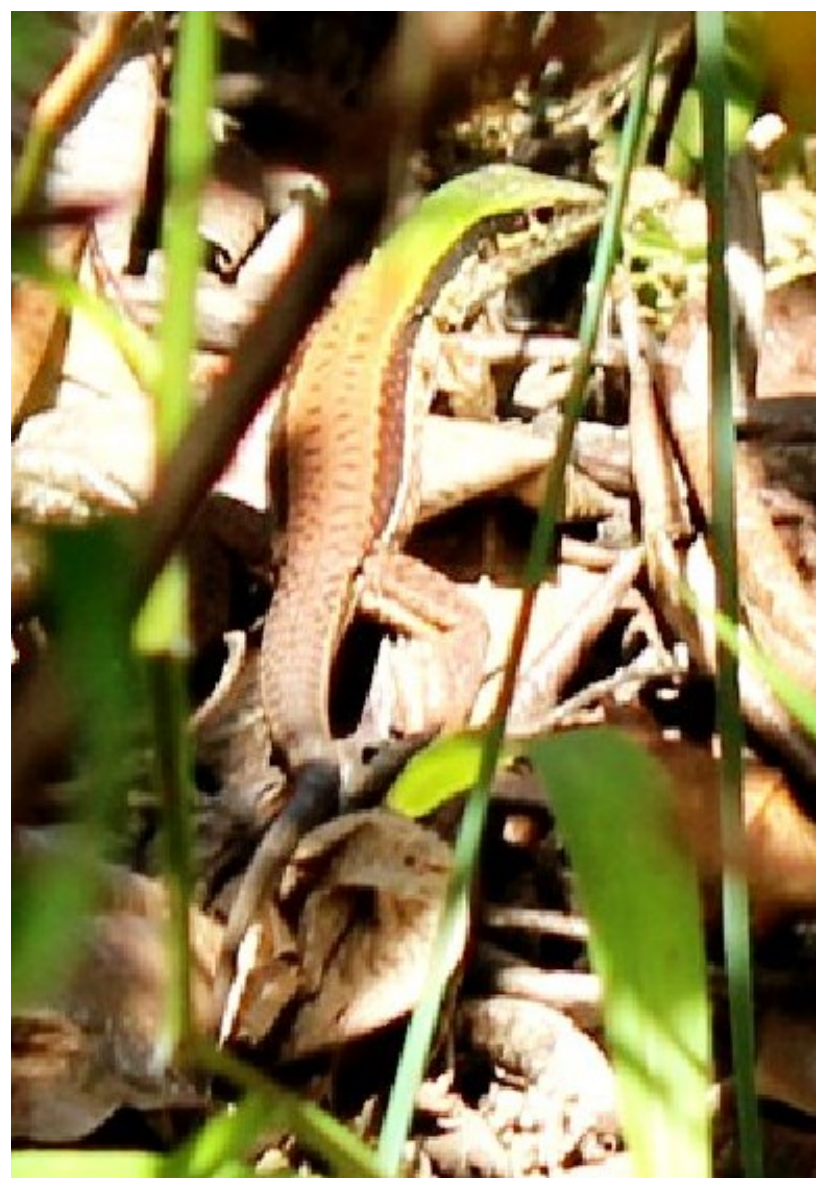

Figura 3. Ameiva ameiva registrado próximo a uma borda de mata. 


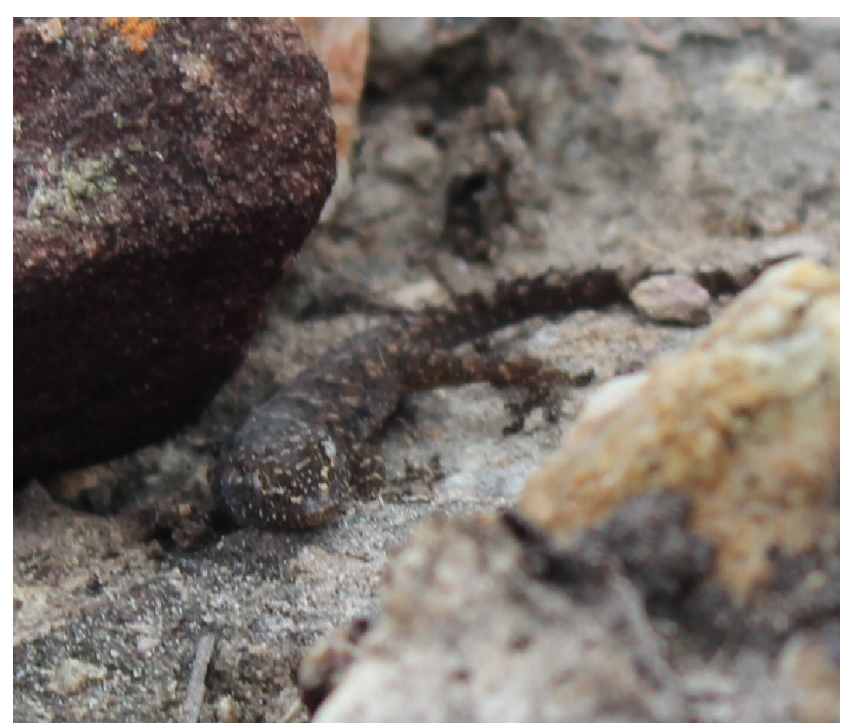

Figura 4. Registro de Hemidactylus palaichthus próximo à rochas.

árvores, sobre troncos de árvores caídas, chão arenoso, cercas e paredes de casas, dentre outros tipos de substrato, como também registrado por Rodrigues (1987).

Ameiva ameiva é geralmente abundante tanto em ambientes naturais quanto antropizados. Tem ampla distribuição geográfica, sendo encontrado em áreas abertas na América do Sul e em Galápagos, no Panamá e em Ilhas do Caribe. É um lagarto terrícola e habita diversos locais como áreas de bordas de mata, clareiras no interior de matas, e em áreas que foram alteradas pelo homem (Andrade 2016). A espécie foi registrada tanto nas armadilhas do ponto 01 e 02 e em seus arredores, quanto em regiões de borda de mata (Figura 3).

\section{Hemidactylus palaichthus apresentou}

hábitos noturnos embora tenham ocorrido registros desses animais também durante o dia nas campanhas de busca ativa. Todos os espécimes estavam ao redor de rochas ou em baixo delas em ambos os períodos do dia (Figura 4). Sua presença já foi registrada nos países da Venezuela, Guyana, Suriname. Esta espécie é encontrada em uma variedade de habitats, incluindo penhascos rochosos, savanas, floresta perene seca e bordas da floresta (Castro \& Mayer 2010).

Ao realizar registros dessa espécie foi possível observar um comportamento de camuflagem. $\mathrm{O}$ animal foi coletado para análise posterior, e percebeu que o mesmo mudou o seu padrão de coloração minutos depois da coleta (Figura 5). Essa adaptação permite que o animal passe despercebido no ambiente, livrando-se de predadores.

\section{CONCLUSÃO}

Os dados obtidos foram corroborados com registros feitos em diferentes regiões da Amazônia e ressaltam que a fauna de lagartos da Fazenda Tepequém assemelha-se aos encontrados em outras regiões da Amazônia. A predominância de registros de Tropidurus hispidus indica um ambiente antropizado. A região do Tepequém apresentou baixa diversidade de espécies. O trabalho exerceu um esforço pontual, mas necessário ao tentar conhecer a composição de lagartos de uma região pouco estudada. Esse resultado pode ser justificado pela fragmentação de hábitat, antropização e pecuária local. Inventários de

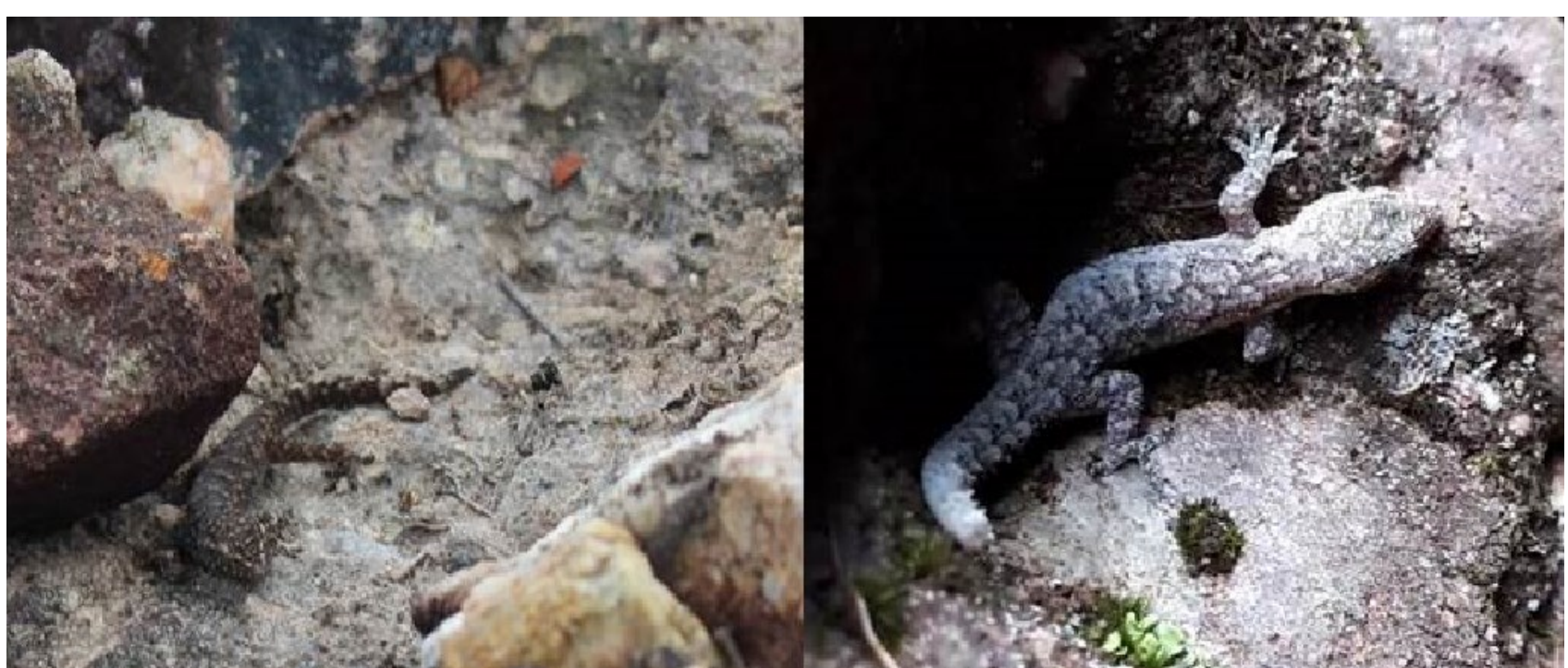

Figura 5. Registro da modificação no padrão de coloração de um espécime de Hemidactylus palaichthus. 
fauna podem indicar lacunas importantes de distribuição de espécies e somente esse tipo de conhecimento pode formar uma base sólida necessária à elaboração de medidas conservacionistas eficientes.

\section{REFERÊNCIAS BIBLIOGRÁFICAS}

Andrade, J. A. 2016. Calango Verde Ameiva ameiva (https://blogdonurof.wordpress.com/2016/02/20/ calango-verde-ameiva-ameiva/). Acesso em 30/08 2016.

Carvalho, M. Celso. 2009. O lavrado da serra da lua em Roraima e perspectivas para estudos da herpetofauna na região. Revista Geográfica Acadêmica 3(1): 4-17.

Carvalho, T.M. \& Carvalho, C.M. 2015. Paisagens e Ecossistemas. In: Silveira, E.D.; Serguei, A.F.C. (Org.). Socioambientalismo de fronteiras: relações homem-ambiente na Amazônia. Ed. Juruá, Curitiba, p. 43-68.

Castro, F. \& Mayer, G.C. 2010. Hemidactylus palaichthus. The IUCN Red List of Threatened Species.

Cechin, S.Z. \& Martins, M. 2000. Eficiência de armadilhas de queda (pitfall traps) em amostragens de anfíbios e répteis no Brasil. Revista brasileira de Zoologia 17(3): 729-740.

Donnelly, M.A., Guyer, G., Juterbock, J.E. \& Alford, R.A. 1994. Techniques for marking amphibians. In Measuring and monitoring biological biodiversity: standard methods for amphibians (W.R. Heyer, M.A. Donnely, R.W. McDiarmid, L.A. Hayek \& M. Foster, eds.). Smithsonian Institution Press, Washington.

Freitas, A.P.P.; Antioro, A.T.B. \& Seabra, D.I. 2017. Eutanásia Hunanitária. Inicamp. Campinas, 1-5.

Macedo, L.M.; Bernarde, P.S. \& Abe, A.S. 2008. Lagartos (Squamata: Lacertilia) em áreas de floresta e de pastagem em Espigão do Oeste, Rondônia, sudoeste da Amazônia, Brasil. Biota Neotropica 8 (1): 133-139.

Mendes-Pinto, T.J.M. \& Tello, J.C.R. 2010. Répteis Squamata de uma área de transição Floresta-Savana no oeste do estado do Pará, Brasil. Revista de Ciências Ambientais 4(1): 19-35.

Monteiro, P.S.D.; Castro, R.B.; Borges-Junior, V.N.; Marra, R.V.; Freitas, R.F.; Amaral, L.B.; Rego, V.C.; Martins-Hatano, F.; Alves, D.N.M. \& Hatano, F.H. 2009. Levantamento de lagartos (Squamata: Lacertilia) na floresta Nacional do Tapirapé - Aquiri, sudeste do Pará, Brasil, MG, São Lourenço, 1-4.

Nascimento, P.S. 2004. Herpetofauna de Roraima: ênfase nas áreas abertas (lavrado). FEMACT, Roraima, 123-137.
Nascimento, S.P. 1998. Ocorrência de Lagartos no "Lavrado" de Roraima (Sauria: Gekkonidae, Teiidae, Iguanidae, Polychridae, Tropiduridae, Scincidae e Amphisbaenidae). Boletim do Museu Integrado de Roraima 4: 39-48.

Rodrigues, M.T. 1987. Sistemática, Ecologia e Zoogeografia dos Tropidurus hispidus do grupo Torquatus ao Sul do Rio Amazonas (Sauria, Iguanidae). Museu de Zoologia da Universidade de São Paulo 31(2): 105-230.

Silva, F.R. \& Rossa-Feres, D.C.. 2007. Uso de fragmentos florestais por anuros (Amphibia) da área aberta na região noroeste do Estado de São Paulo. Biota Neotropica 7(2): 141-148. 


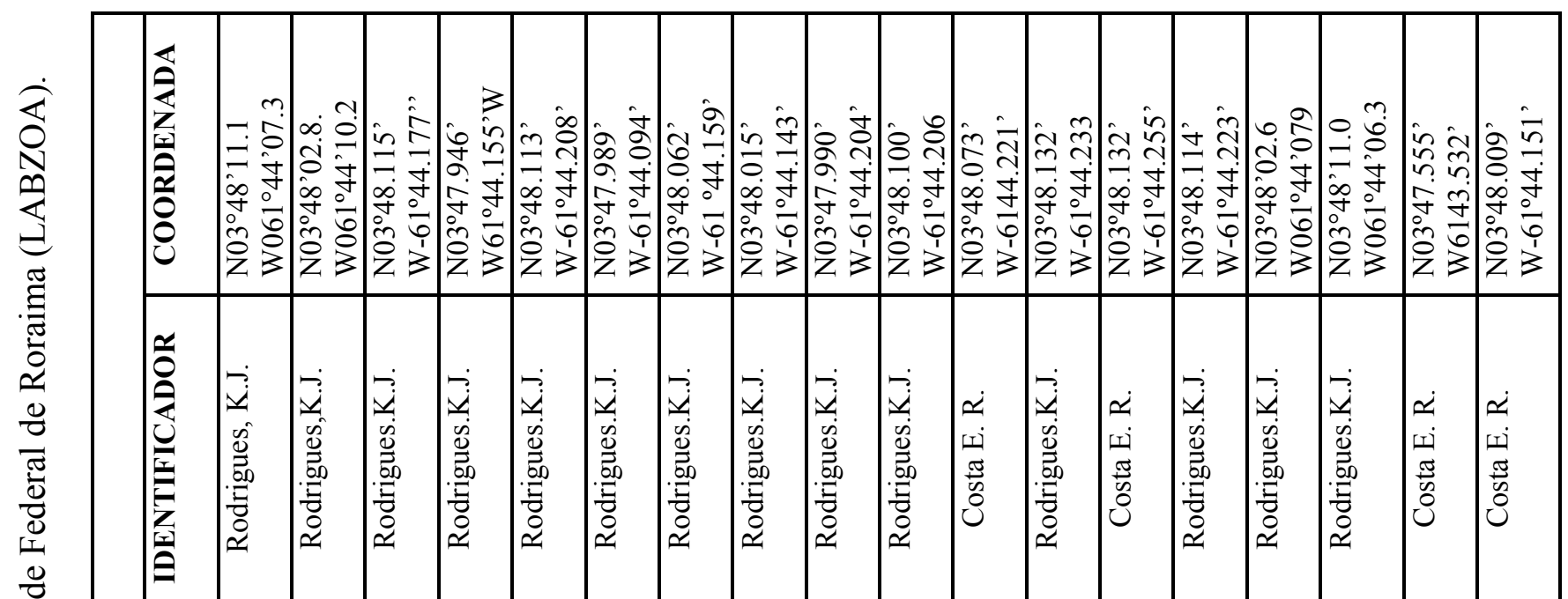

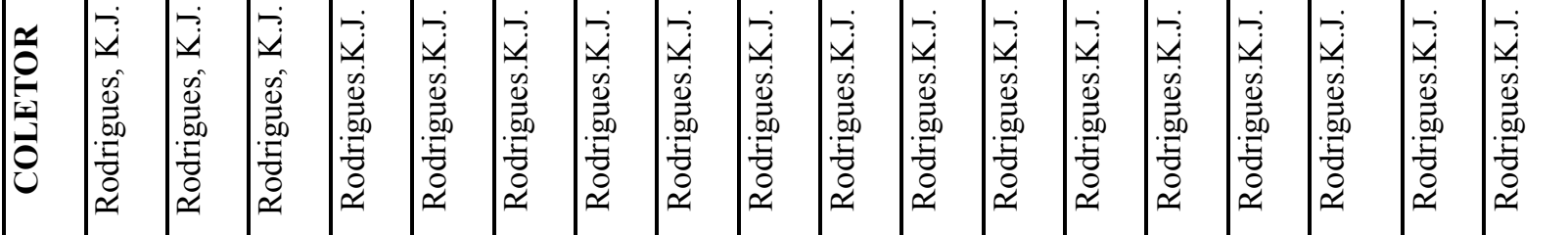



N

\begin{tabular}{|c|c|c|c|c|c|c|c|c|c|c|c|c|c|c|c|c|c|}
\hline 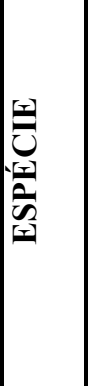 & 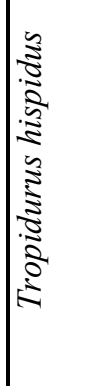 & 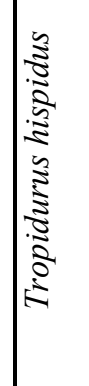 & 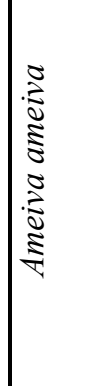 & 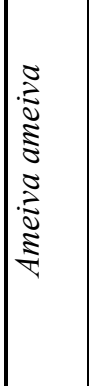 & 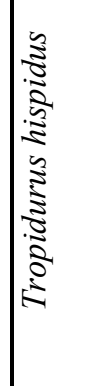 & 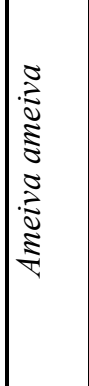 & 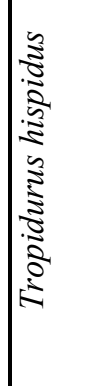 & 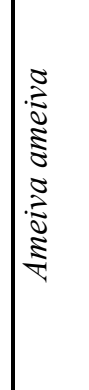 & 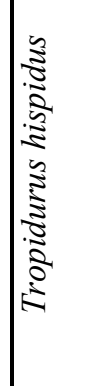 & 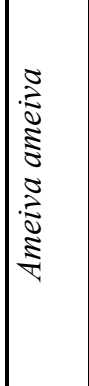 & 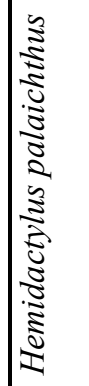 & 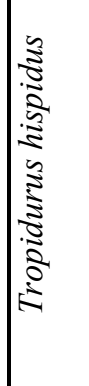 & 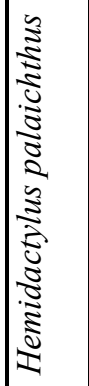 & 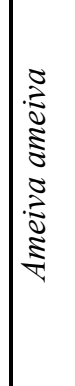 & 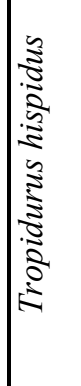 & 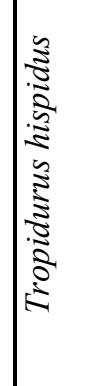 & 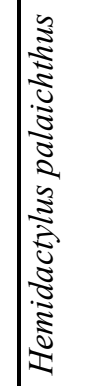 \\
\hline 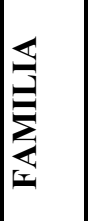 & 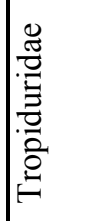 & 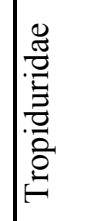 & $\frac{\mathbb{\pi}}{:}$ & 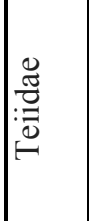 & 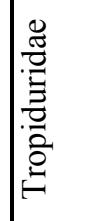 & 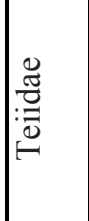 & 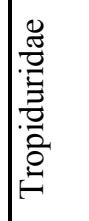 & 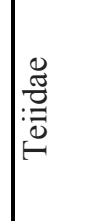 & 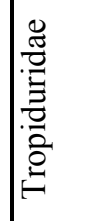 & $\frac{\mathbb{\pi}}{:}$ & 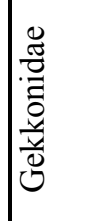 & 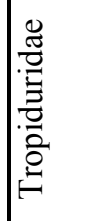 & 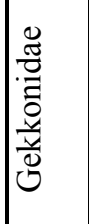 & 兽 & 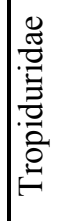 & 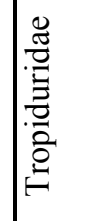 & 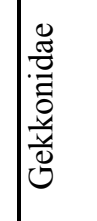 \\
\hline 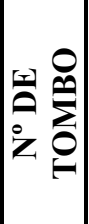 & 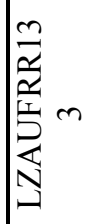 & 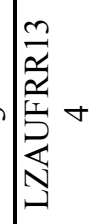 & 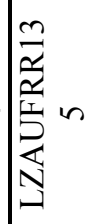 & 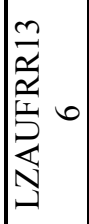 & 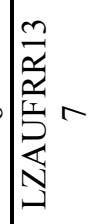 & 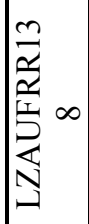 & 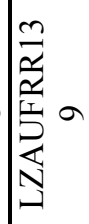 & 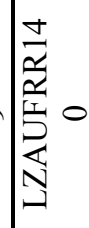 & 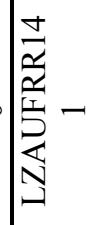 & 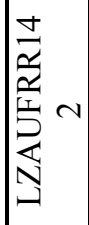 & 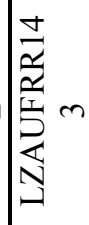 & 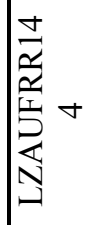 & 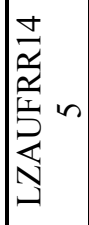 & 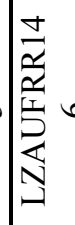 & 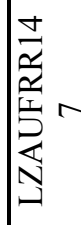 & 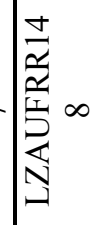 & 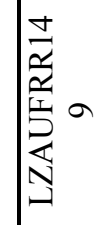 \\
\hline
\end{tabular}

